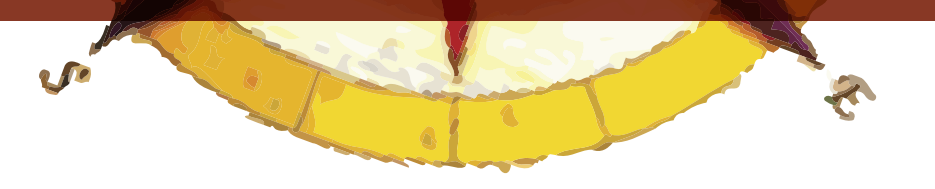

\title{
MUCHOS NÚMEROS Y POCOS PROBLEMAS
}

\author{
Ana María Ribes y Xosé M. Souto ${ }^{1}$
}

\section{RESUMEN}

En el sistema escolar los estudios de la población suelen representar un panorama descriptivo y estadístico de las principales magnitudes demográficas: densidades, nacimientos, defunciones, población activa, migraciones, distribución por cohortes de edad. Sin embargo son menores las referencias a los problemas cotidianos de las personas en sus diferentes lugares geográficos. El estudio de la población activa y el mundo del trabajo muestra este alejamiento entre los conceptos académicos y los problemas cotidianos.

Palabras Clave: geografía de la población, población activa, trabajo, problemas sociales.

\section{RESUMO}

No sistema escolar, os estudos da população apenas apresentam um panorama descritivo e estatístico das principais variáveis demográficas: densidades, nascimentos, mortalidades, população ativa, migrações, distribuição por grupos etários. Ao contrario, são menores as referências aos problemas quotidianos das pessoas nos seus diferentes lugares geográficos. $O$ estudo da população ativa e o mundo do trabalho mostra este afastamento entre os conceitos académicos e os problemas quotidianos.

Palavras Chave: geografia da população, população ativa, trabalho, problemas sociais.

\begin{abstract}
In the school system population studies often represent a descriptive and statistical overview of the main demographic variables: density, births, deaths, active population, migration, distribution
\end{abstract}

1 Universitat de València, Geoforo Iberoamericano de Educación 
by age cohorts. However, there are minor references to the everyday problems of people in different geographical locations. The study of the active population and the world of work show this separation between academic concepts and everyday problems.

Keywords: geography of population, active population, employment, social problems.

\section{INTRODUCCIÓN}

La concepción de la geografía de la población está inserta en una manera de entender las situaciones demográficas donde predomina una cuantificación en aras de una pretendida objetividad del estudio. En este sentido la geografía escolar aborda la descripción de la distribución de las diferencias, pero no se cuestiona la explicación de los factores de localización de las desigualdades.

El estudio de un caso concreto, como es el análisis del mundo del trabajo desde las teorías de la población activa nos permitirá comprobar hasta qué punto los conceptos que se utilizan no responden a las expectativas sociales del alumnado, como también sucede en los docentes.

El artículo se organiza así en dos partes fundamentales. En una primera damos cuenta de la evolución de los estudios de geografía de la población en España desde 1950, con sus programas oficiales y las aplicaciones que hacían los manuales escolares de ellos. Con ello se creaban unas rutinas y hábitos escolares que han llegado hasta el siglo XXI, pese a algunos intentos de renovación2. Después en una segunda parte damos cuenta de una investigación sobre la geografía de la población activa que forma parte de un amplio proyecto de estudio iberoamericano; con este estudio de caso pretendemos acercar la impugnación teórica de los conceptos académicos a la realidad del aula de los centros escolares de enseñanza básica. Entendemos que esta metodología es la que nos permitirá acercar la investigación teórica universitaria a la realidad práctica de las aulas con el universo demográfico de los niños, jóvenes y adolescentes.

\section{LA ENSEÑANZA DE LA GEOGRAFÍA DE LA POBLACIÓN DESDE 1950 EN ESPAÑA}

Para entender la evolución histórica de la geografía de la población en el sistema escolar vamos analizar de forma complementaria dos elementos que nos permiten verificar sus dinámicas, hábitos y rutinas. Por una parte, la evolución de los programas oficiales emanados de las leyes educativas; es una propuesta de las autoridades administrativas y que se corresponde en cierta medida con la evolución del pensamiento geográfico institucional. Por la otra vamos a realizar el estudio de los manuales escolares para verificar cómo se traslada a las rutinas de aula las propuestas académicas.

\section{El currículo oficial. De las leyes a los temarios}

Podemos explicar la dinámica legislativa de la geografía escolar a partir de la mitad del siglo XX como el resultado de un esfuerzo institucional por definir un cuerpo disciplinar de hechos y conceptos que permitiera superar en los años cincuenta la propuesta ideológica que surgía del plan de 
1938, inspirado en la retórica fascista de los sublevados contra el orden constitucional de la Segunda República.

Centrándonos en el caso de la Educación Secundaria nos encontramos en el franquismo con la Ley de Ordenación de la Enseñanza Media de 26 de febrero de 1953, que sustituye la Ley de 1938. En los años cincuenta la apertura de España (reconocimiento por el Vaticano, bases militares de Estados Unidos en España y entrada en la ONU) coincide con la llegada de los tecnócratas del Opus Dei a las altas instancias de educación.

En el caso de la enseñanza de la geografía la figura de Pedro Plans, catedrático en Murcia y director de la revista Didáctica Geográfica, es relevante para entender este giro "modernizador" de la enseñanza de la geografía en cuanto a los conceptos. Ello se puede comprobar en el programa de 1957 según Orden de 4 de junio de 1957 sobre cuestionarios y textos para el Bachillerato (B.M. 1/ VIII/1957) que hace referencia al Decreto de 31 de mayo de 1957.

La Geografía aparecía como Geografía de España en $1^{\circ}$ de Bachillerato elemental y en Geografía General en $2^{\circ}$ Bachillerato elemental. O sea, para edades comprendidas entre los diez y doce años. La concepción curricular era claramente positivista e instructiva: se entendía que el alumno aprendería los hechos y conceptos que aparecían en un temario y que era desarrollado por escrito en los libros de texto y de forma oral por las explicaciones docentes de los libros de texto.

Desde estos años se han sucedido las leyes educativas y los temarios de hechos y conceptos donde aparecía el contenido de la geografía de la población. El cuadro 1 refleja las principales propuestas de contenidos didácticos que hacen referencia a la geografía de la población. En dicho cuadro podemos ver cómo ha evolucionado la concepción de la Geografía de la Población, desde un planteamiento más cercano al estudio de los géneros de vida ("cuasi" etnografía) hasta un estudio mucho más estadístico y pretendidamente objetivo en el que se pretende analizar el problema de la población entendido como el crecimiento demográfico malthusiano. Ello, como se comentará, está relacionado con el cambio de modelo pedagógico del sistema escolar, donde el modelo tradicional es sustituido por un sistema tecnocrático.

Cuadro 1. Leyes educativas y temas de geografía de la población

\begin{tabular}{|l|l|l|l|}
\hline Leyes educativas & Decreto/Orden & \multicolumn{1}{|c|}{ Organización curricular } & \multicolumn{1}{|c|}{$\begin{array}{c}\text { Temas. Contenidos de } \\
\text { población }\end{array}$} \\
\hline Ley de 1953 & 1957 (mayo/junio) & $\begin{array}{l}\text { Geografía en } 1^{\circ} \text { y } 2^{\circ} \\
\text { Bachillerato Elemental: } 11-12 \\
\text { años de edad. España en } 1^{\circ} \text { y } \\
\text { general en } 2^{\circ}\end{array}$ & $\begin{array}{l}\text { La población y el } \\
\text { poblamiento en España }\left(1^{\circ}\right) . \\
\text { El hombre y el medio. La } \\
\text { población mundial }\left(2^{\circ}\right)\end{array}$ \\
\hline Ley de 1953 & 1967 (mayo) & $\begin{array}{l}\text { Igual que en el caso anterior: } \\
1^{\circ} \text { y } 2^{\circ} \text { de Bachillerato } \\
\text { elemental }\end{array}$ & $\begin{array}{l}\text { La población española. } \\
\text { El campo y la ciudad. El } \\
\text { hombre y el medio geográfico }\end{array}$ \\
\hline
\end{tabular}




\begin{tabular}{|c|c|c|c|}
\hline Ley de 1970 & 1971 (agosto) & $\begin{array}{l}\text { Ciclo medio y superior de } \\
\text { EGB: } 9 \text { a } 14 \text { años de edad. } \\
\text { Programación por círculos } \\
\text { concéntricos: localidad, } \\
\text { región, España y Mundo }\end{array}$ & $\begin{array}{l}\text { Aparecen trabajos con } \\
\text { estadísticas. Objetivos } \\
\text { operativos. El crecimiento de } \\
\text { la población. Migración }\end{array}$ \\
\hline $\begin{array}{l}\text { Ley de 1970: } \\
\text { Ley General de } \\
\text { Educación }\end{array}$ & 1975 (marzo) & $\begin{array}{l}2^{\circ} \text { de Bachillerato Unificado } \\
\text { y Polivalente: } 16 \text { años de } \\
\text { edad (Geografía Humana y } \\
\text { Económica) }\end{array}$ & $\begin{array}{l}\text { Geografía de la población } \\
\text { La población mundial: } \\
\text { efectivos y distribución } \\
\text { Movimientos naturales y } \\
\text { migratorios } \\
\text { Problemática del crecimiento } \\
\text { actual de la población }\end{array}$ \\
\hline LOGSE de 1990 & $\begin{array}{l}\text { Real Decreto } \\
1007 / 1991\end{array}$ & $\begin{array}{l}\text { Organización por ciclos en la } \\
\text { ESO (12 a } 16 \text { años): en ambos } \\
\text { ciclos aparecen contenidos } \\
\text { de Geografía. También en } 2^{\circ} \\
\text { Bac. ( } 18 \text { años): Geografía de } \\
\text { España }\end{array}$ & $\begin{array}{l}\text { Bloque Sociedad y Territorio: } \\
\text { la población y los recursos; } \\
\text { actividades económicas y el } \\
\text { espacio geográfico }\end{array}$ \\
\hline LOCE & $\begin{array}{l}\text { Real Decreto } \\
3473 / 2000\end{array}$ & $\begin{array}{l}\text { Los contenidos se organizan } \\
\text { por cursos. Geografía en } 1^{\circ} \text {, } \\
2^{\circ} \text { y } 3^{\circ} \text { de ESO y en } 2^{\circ} \text { Bach. }\end{array}$ & La población ( $\left.2^{\circ} \mathrm{ESO}\right)$ \\
\hline LOE & $\begin{array}{l}\text { Real Decreto } \\
1631 / 2006, \text { de } 29 \\
\text { de diciembre, }\end{array}$ & $\begin{array}{l}\text { Contenidos por cursos. La } \\
\text { Geografía aparece en } 1^{\circ}, 2^{\circ} \text { y } \\
3^{\circ} \text { de ESO y en } 2^{\circ} \text { Bach. }\end{array}$ & La población ( $\left.2^{\circ} \mathrm{ESO}\right)$ \\
\hline
\end{tabular}

Fuente: Elaboración propias sobre Reales Decretos de enseñanzas mínimas.

Como podemos observar desde 1957 hasta 1975 predomina una selección de contenidos que hace referencia a un temario de hechos y conceptos en los cuales la población aparece como un elemento integrado en un medio geográfico, definido por su dinámica y su organización territorial, dentro de la lógica del paradigma regional, en el cual la acción antrópica desarrolla las posibilidades de los recursos del medio.

La ampliación de los estudios básicos y obligatorios hasta los catorce años supuso la programación de unos nuevos contenidos educativos. Estos se programaron de una forma técnica y pretendidamente eficientes: los objetivos operativos. En el caso de la geografía de la población ésta aparece ya en tercero de EGB (8 años de edad) con unos contenidos referidos a la dinámica de la población que se expresaba de la siguiente manera:

Analizar el índice de crecimiento de una población relacionándolo con el fenómeno de las migraciones. 
Después en cuarto y quinto curso se aplica este principio a la región donde vive el alumno y al conjunto de España, con enunciados que nos remiten a localizar las zonas más pobladas y a construir e interpretar gráficos. Es decir, la variante técnica de los objetivos operativos se complementaba con una propuesta de estudio instrumental de trabajo demográfico. Por último en octavo de EGB (14 años de edad) se programa un estudio de la población del mundo por grandes regiones paisajísticas y de desarrollo económico. En la sección de Geografía de España la población es un soporte estadístico para ver las desigualdades territoriales.

En 1975 se establece por normativa legal el contenido de Geografía Humana y Económica; adquiriendo un peso significativo el estudio de la población, pues era la manera frecuente de iniciar el estudio de los contenidos de esta materia escolar. Todo se organizaba desde la dinámica de la población, que se concretaba en el espacio mundial de acuerdo a la triple división territorial: sistemas capitalistas, socialistas y Tercer Mundo. La visión de la geografía como género de vida estaba impugnada por los recientes debates en la geografía cuantitativa y surgía como modelo esqueuomórfico una descripción estadística de la dinámica y estructura demográfica. Además se reforzaba la idea de la geografía escolar como materia de ciencias sociales, en gran medida como consecuencia de la organización administrativa de áreas de conocimiento con la LGE: el área social y antropológica.

La llegada de la democracia y el debate sobre los contenidos escolares incide en la promulgación de la LODE en los años ochenta y en la LOGSE de los años noventa, que presenta una concepción de contenidos escolares que destaca con más hincapié el papel de los procedimientos para alcanzar la construcción de hechos y conceptos y el desarrollo de actitudes que favorecería la creación de hábitos, valores y responsabilidades éticas. Una modificación en los objetivos que era congruente con los cambios que se operaban en la geografía y demografía académica ${ }^{3}$, de tal manera que se presuponía lo que se vino en denominar trasposición didáctica; o sea la traslación de los conceptos académicos a las aulas del sistema escolar básico.

Así en la LOGSE se pretende relacionar a la población con su medio ecológico, tal como se desprende del título sociedad y territorio y del enunciado de los bloques de contenidos que se organizaban de una forma flexible por ciclos educativos. Será en los momentos previos a la aprobación de dicha Ley cuando aparezcan diversos movimientos pedagógicos que intentan desarrollar alternativas didácticas en el estudio geográfico de la población. La metodología activa se solía confundir con una utilización de un número elevado de técnicas y recursos didácticos: elaboración de gráficas, comentarios de mapas, dossier sobre problemas del crecimiento de la población...

Este viraje hacia un análisis de la dinámica y estructura de la población, además del incremento coyuntural de recursos y tareas sobre los movimientos migratorios va a orientar los estudios de geografía de la población. Es decir, de una descripción de géneros de vida se continúa con un estudio de la población como problema de crecimiento y finalmente se desarrollan temas-problema, como es el caso de las migraciones.

Las continuas transformaciones del sistema escolar entre 1991 y 2014 han provocado una sensación de incertidumbre por parte del profesorado, que además veía como en cada reforma sucesiva se "cerraban" más los contenidos. Es decir, se volvía a una lógica de temario de contenidos conceptuales. Ello es bien evidente en el caso de la geografía de la población, pues del enunciado de

3 En este sentido podemos consultar los trabajos de R. Puyol (1996 y 1997) y de A. Izquierdo (2003). 
"La población y los recursos" del año 1991 con una organización flexible que se debía adaptar a las características sociales del centro escolar y a la autonomía profesional del docente, se pasa a la delimitación de "La población mundial" como un conjunto de hechos y conceptos que se deben impartir en segundo de ESO (13 años de edad) $)^{4}$.

Cuando analizamos las concreciones del currículo, en el caso de los decretos de las Comunidades Autónomas, observamos que los cambios tienen todavía mayor incidencia en la práctica escolar. La comparación de los decretos de Galicia ${ }^{5}$ de la Comunidad Valenciana ${ }^{6}$ nos permiten comprobar el cierre conceptual y factual de los contenidos de la geografía de la población que refuerza esa visión aséptica y neutra de la dinámica y estructura de la población. Así frente a la presentación de un contenido de Distribución de las desigualdades socioeconómicas en la tierra aparece otro titulado simplemente La población mundial, que da lugar a todo un repertorio de estadísticas y conceptos que desarrollan los libros de texto, como vamos a analizar.

De tal manera que en la LOE del año 2007 los contenidos de Geografía de la Población se expresan de esta manera para $2^{\circ}$ curso de la Educación Secundaria Obligatoria (13 años de edad $)^{7}$ :

\section{Bloque 2. Población y sociedad}

La población.

- Crecimiento, regímenes demográficos, movimientos migratorios y distribución.

- Aplicación de los conceptos básicos de demografía a la comprensión de las tendencias actuales del crecimiento de la población. Análisis y valoración de sus consecuencias en el mundo y en España, con referencia específica a la Comunitat Valenciana.

- Las estructuras demográficas. Diferencias según niveles y modelos de desarrollo

- La población española. Evolución y distribución. Contrastes regionales. La población de la Comunitat Valenciana.

Como se puede apreciar sigue existiendo una manera de organizar los contenidos que siguen la tendencia que se había iniciado con la LGE de 1970, si bien en estos momentos los contenidos propios de la Comunidad Autónoma (La Comunitat Valenciana) están más destacados.

El currículo real: los contenidos en los manuales escolares

Los manuales escolares aplican el contenido según normas establecidas por las administraciones. Además generan unas rutinas metodológicas que muchas veces se consideran como "naturales". Es decir, docentes y alumnos entienden que la manera de presentar los contenidos en un manual escolar es la forma única y natural de explicarlos y presentarlos en clase para facilitar su aprendi-

$4 \quad$ Ver Reales Decretos 1007/1991, de 14 de junio, y 3473/2000 de 29 de diciembre

5 Decretos de la Xunta de Galicia: 78/1993 de 25 de febrero y 233/2002, de 6 de junio

6 Decretos de la Generalitat Valenciana: 47/1992, de 30 de marzo y 39/2002 de 5 de marzo.

7 Utilizamos como referencia el DECRETO 112/2007, de 20 de julio, del Consell, por el que se establece el currículo de la Educación Secundaria Obligatoria en la Comunitat Valenciana. 
zaje. Para ello los redactores de los manuales buscan organizar los contenidos en relación directa con los cambios legislativos y las aportaciones de la materia disciplinar, en este caso geografía de la población. Además hemos de saber que la censura administrativa ha condicionado la elaboración de los temas y ello ha generado una rutina que ha afectado a la autocensura, como se comentará.

En el contexto de la Dictadura franquista se habían creado instituciones para regular y controlar la edición de textos escolares ${ }^{8}$. Posteriormente la Ley de Educación Primaria de 1945 recoge en su articulado la necesidad de autorización previa de los libros de texto ${ }^{9}$, que se confirma en el Decreto de 22 de septiembre de 1955 y en el Decreto de 21 de marzo de 1958, que afectaba a Primaria y Enseñanza Media ${ }^{10}$. Un control ideológico que todavía permanecía en los años setenta ${ }^{11}$.

Pero posteriormente los libros de texto cambiaron de normativa, de tal manera que primero sólo se revisaba el conjunto del proyecto curricular y no los contenidos precisos de cada unidad didáctica $^{12}$, desapareciendo la autorización previa en 1998, imponiéndose un régimen jurídico en el que dicha supervisión no revestía el carácter de previa ${ }^{13}$, sino que se sometía a los códigos civil y penal.

Por tanto cabe preguntarse: ¿Por qué en los libros de texto de los años finales de siglo XX y en el primer decenio del siglo XXI sigue predominando una visión homogénea de la población mundial? ¿Por qué no hay más diferencias en los enfoques teóricos y metodológicos? ¿Cómo influyen las rutinas que se han fraguado en los últimos años del franquismo? ¿Cuál es el peso efectivo de la trasposición didáctica desde el conocimiento científico?

Una hipótesis de trabajo que planteamos consiste en subrayar que la concepción educativa de la geografía como una ciencia en singular, algo que ya combatimos en otra ocasión (Souto, 2010). Es decir, se consideraba que los contenidos de Geografía de la Población respondían a lo que se estaba haciendo en la investigación geográfica y que, por tanto, no era posible organizarlos de otra manera.

Así una definición de geografía de la población que se acepta como única decía lo siguiente:

La Geografía de la población estudia la población en relación con el espacio. En esto se diferencia de otras ciencias (Demografía, Etnología) que también se ocupan del estudio de la población.

Conceptos básicos para el estudio de la Geografía de la población son la población absoluta, la densidad media y la superpoblación.

$8 \quad$ Es el caso del Instituto de España que desde 1938 tenía encomendada la misión de editar los libros de texto de Educación Primaria que se imponían con carácter obligatorio.

9 En su artículo 48 indicaba que "los libros de uso escolar en todas las escuelas españolas habrán de ser aprobados por el Ministerio de Educación y Ciencia, previos los asesoramientos técnicos en cuanto a su contenido y confección, sin lo cual no podrá utilizarse en la primera enseñanza ni como textos ni como libros de lectura". Entre las referencias doctrinarias que debían seguirse estaba el Título I de la presente Ley que destacaba el amor a la patria bajo los auspicios del Movimiento nacional (o sea, fascista). Ley de Educación Primaria de 17 de Julio de 1945.

$10 \quad$ BOE de 1 y 15 de abril de 1958

11 Es el caso del Decreto 2531/1974, de 20 de julio, sobre autorizaciones de libros de texto y material didáctico. (BOE de 13 de septiembre de 1974).

12 Real Decreto de 15 de abril de 1992, BOE 23 de abril de 1992

13 Boletín Oficial del Estado de 4 de septiembre de 1998 
La población mundial supera los cinco mil millones de personas, cifra a la que se ha llegado mediante un lento progreso a través de miles de años.

Este progreso se ha acelerado de forma muy rápida en las últimas décadas, por lo que hoy puede hablarse de que existe una verdadera explosión demográfica ${ }^{14}$.

De esta manera los contenidos de Geografía de la población como contenidos de autor reflejaban una secuencia que empezaba en la problemática de la población mundial en su dinámica demográfica, después continuaba en los factores que determinaban los movimientos naturales y migratorios, para analizar a continuación la distribución de la población por sexo, edad y actividad económica y, por último, describir los rasgos espaciales de los tres sistemas geoeconómicos mundiales, si bien en el caso de la Educación General Básica los espacios considerados son las Comunidades Autónomas y España. En el cuadro 2 podemos ver algunos ejemplos de lo que decimos.

Cuadro 2. Contenidos de geografía de la población en los manuales escolares

\begin{tabular}{|l|l|l|}
\hline $\begin{array}{c}\text { Curso escolar y edad } \\
\text { del alumnado }\end{array}$ & Libros analizados (códigos) & \multicolumn{1}{|c|}{$\begin{array}{c}\text { Contenidos de geografía de la } \\
\text { población }\end{array}$} \\
\hline $1^{\text {o }}$ Bach (11 años) & 1.GRIMA, 1963 & $\begin{array}{l}\text { lección 36 expone: } \\
\text { Población. La población actual. Su } \\
\text { distribución: zonas de concentración y } \\
\text { de repulsión de la población. Densidad } \\
\text { de la población. Las diferencias } \\
\text { regionales: idiomas y dialectos. } \\
\text { Expansión del castellano en el mundo. } \\
\text { La Religión }\end{array}$ \\
\hline $1^{\circ}$ Bach (11 años) & 2.COMAS, 1968 & $\begin{array}{l}\text { El crecimiento constante de la } \\
\text { población y el desigual reparto de la } \\
\text { población. }\end{array}$ \\
\hline $1^{\circ}$ Bach (11 años) & 3. RASTRILLA, 1968 & $\begin{array}{l}\text { Texto lleno de tópicos (lección 9) } \\
\text { La población se caracteriza por } \\
\text { "el individualismo y el espíritu } \\
\text { de independencia; su sobriedad y } \\
\text { resistencia física; por el valor y el } \\
\text { heró́smo; la religiosidad y la lealtad" } \\
\text { (sic, página 67) }\end{array}$ \\
\hline $2^{\circ}$ Bach. (12 años) & 4.ASIÁN PEÑA, 1968 & $\begin{array}{l}\text { La lección 9, el hombre y el medio } \\
\text { geográfico (páginas 68-75) habla de la } \\
\text { población y el Poblamiento }\end{array}$ \\
\hline $8^{\circ}$ EGB (14 años) & 5.VERGES, 1974 & \begin{tabular}{l} 
Fichas demográficas para hacer gráficas \\
\hline
\end{tabular} \\
\hline
\end{tabular}

14 ALVARES OSES, José A.; SABÁN GUTIÉRREZ, A.; MARTÍN REDONDO, G. Geografía humana y económica, Madrid: Santillana, 1987, pàgina 4 


\begin{tabular}{|c|c|c|}
\hline $6^{\circ}$ EGB (12 años) & 6. BORRAS, M. et al., 1983 & $\begin{array}{l}\text { La población mundial. Ecúmene } \\
\text { y distribución de la población. } \\
\text { Crecimiento. Natalidad. Migración } \\
\text { Mortalidad. Población activa. }\end{array}$ \\
\hline $2^{\circ}$ BUP (16 años) & 7. GARBI, 1984 & $\begin{array}{l}\text { *la población mundial } \\
\text { *la estructura de la población } \\
\text { *polémica sobre la población } \\
\text { *Las fuentes demográficas }\end{array}$ \\
\hline $2^{\circ}$ BUP (16 años) & 8.DIDESPAI, 1986 & $\begin{array}{l}\text { Evolución de la población; regímenes } \\
\text { demográficos; dimensión espacial de } \\
\text { los fenómenos demográficos; debates } \\
\text { demográficos; estructura por edades y } \\
\text { sexo. }\end{array}$ \\
\hline $2^{\circ}$ BUP (16 años) & 9.EDETANIA, 1986 & $\begin{array}{l}\text { La población aparece entre las páginas } \\
37 \text { y } 82 \text {. Se estructura en cinco grandes } \\
\text { apartados: Fuentes de la información, } \\
\text { evolución y distribución de la población } \\
\text { mundial, estructura de la población, } \\
\text { problemática del crecimiento de la } \\
\text { población }\end{array}$ \\
\hline $2^{\circ}$ BUP (16 años) & 10.ÁVAREZ OSES, 1987 & $\begin{array}{l}\text { Los cuatro temas de la población son: } \\
\text { 1.-Efectivos y distribución de la } \\
\text { población mundial } \\
\text { 2.-Movimientos naturales de la } \\
\text { población } \\
\text { 3.-Movimientos migratorios } \\
\text { 4.-La estructura de la población } \\
\text { (edades, sexo, profesiones, lenguas, } \\
\text { razas, religiones) }\end{array}$ \\
\hline $6^{\circ}$ EGB (12 años) & 11.FERNÁNDEZ, A et al., 1988 & $\begin{array}{l}\text { La población. Distribución por zonas } \\
\text { bioclimáticas. Dinámica. La población } \\
\text { actual. Del campo a la ciudad. }\end{array}$ \\
\hline $6^{\circ}$ EGB (12 años) & $\begin{array}{l}\text { 12.PERALES, J. y PELEGERO, V., } \\
1990\end{array}$ & $\begin{array}{l}\text { Efectivos de población, factores } \\
\text { demográficos, población en Valencia y } \\
\text { España. Problemas demográficos. }\end{array}$ \\
\hline
\end{tabular}




\begin{tabular}{|l|l|l|}
\hline & & $\begin{array}{l}\text { La población aparece en: } \\
\text { 3.-La población y el desarrollo humano, } \\
\text { con cuatro epígrafes: la desigual } \\
\text { distribución de la población, dinámica } \\
\text { natural y estructura demográfica, } \\
\text { los movimientos migratorios, la } \\
\text { distribución de la riqueza }\end{array}$ \\
$\begin{array}{l}2^{\circ} \text { ciclo ESO (14-16 } \\
\text { años de edad) }\end{array}$ & 13. MOLINERO et al, 1998 & $\begin{array}{l}\text { 17.Población y poblamiento (en } \\
\text { España) con la distribución espacial y } \\
\text { la dinámica natural; el sistema urbano } \\
\text { yestructura interna, el poblamiento } \\
\text { rural; infraestructuras de transporte y } \\
\text { accesibilidad. }\end{array}$ \\
\hline $2^{\circ}$ curso (13 años) & 14. FENOSA, 2010 & $\begin{array}{l}\text { Densidad, evolución de la población, } \\
\text { estructura, migraciones, población } \\
\text { activa y aplicación al caso de España }\end{array}$ \\
\hline $3^{\circ}$ de ESO & $\begin{array}{l}\text { La población se desarrolla en tres } \\
\text { unidades: } 1 .- \text { población y tensiones } \\
\text { demográficas: población mundial y } \\
\text { española (distribución, movimientos } \\
\text { naturales y migratorios, estructura); } \\
\text { trabajo de técnicas (elaborar y comentar } \\
\text { pirámides de población). } \\
\text { 2.- sociedades actuales y sus diferencias } \\
\text { (evolución y rasgos culturales, } \\
\text { económicos, sociales y políticos), } \\
\text { transformaciones de la sociedad } \\
\text { europea y española actual. } \\
\text { 3.- el poblamiento humano en las } \\
\text { ciudades. }\end{array}$ \\
\hline 15. BURGOS ALONSO, 2012
\end{tabular}




\begin{tabular}{|c|c|c|}
\hline $\begin{array}{l}2^{o} \text { de ESO (12-13 años } \\
\text { de edad) }\end{array}$ & 16. BRANDI FERNÁNDEZ, 2012 & $\begin{array}{l}\text { Estudio de la población y la vida } \\
\text { en sociedad desarrollada en tres } \\
\text { unidades: 1.- población mundial } \\
\text { (distribución de la población, natalidad, } \\
\text { mortalidad, esperanza de vida, políticas } \\
\text { demográficas y migraciones); trabajo } \\
\text { de técnicas (pirámides de población, } \\
\text { estadísticas de tasas demográficas, } \\
\text { gráficos lineales y de barras, uso de la } \\
\text { web INE). } \\
\text { 2.- población diversa (sociedades según } \\
\text { estructura económica, diversidad } \\
\text { cultural, desigualdades y conflictos; } \\
\text { trabajo de técnicas (elaboración e } \\
\text { interpretación de gráficos sectoriales, } \\
\text { encuestas). } \\
\text { 3.- población y sociedad en la UE, } \\
\text { España y Comunidad Valenciana } \\
\text { (población, migraciones, problemas } \\
\text { sociales); trabajo de técnicas } \\
\text { (interpretación mapa coropletas). }\end{array}$ \\
\hline
\end{tabular}

Fuente: Elaboración propia sobre manuales escolares indicados en REFERENCIAS

Para analizar más adecuadamente los libros de texto en Geografía de la Población vamos a seguir el estudio realizado en su día por la profesora Teresa Benedito ${ }^{15}$, donde mostraba las rutinas y estereotipos que se repetían en los diferentes cursos de Educación General Básica y de Bachillerato (ver cuadro 3). Las fuentes utilizadas en dicho trabajo fueron las siguientes ${ }^{16}$ :

Cuadro 3. Comparación de manuales de Geografía. El estudio de la población.

\begin{tabular}{|l|l|}
\hline \multicolumn{1}{|c|}{ Manuales de EGB (12 años edad) } & \multicolumn{1}{c|}{ Manuales BUP (16 años edad) } \\
\hline Sociedad, edit. Santillana, 1983 (34-41) & Geografía, ECIR, 1989, pp. 32-71 \\
\hline $\begin{array}{l}\text { Coneixement del medi: Espanya i Europa, } \\
\text { Santillana, 1995, pp. 14-17 }\end{array}$ & $\begin{array}{l}\text { Geografía humana y económica del mundo actual. } \\
\text { Intercambio, Vicens Vives, 1988, pp. 2-31 }\end{array}$ \\
\hline Socials, Edebé, 1993, págs.. 58-66 & $\begin{array}{l}\text { Geografía Humana y Económica, Santillana, } \\
\text { 1987, pp. 4-45 }\end{array}$ \\
\hline Nuevo País, Vicens vives, 1987; pp. 40-51 & \multicolumn{1}{|c|}{. } \\
\hline
\end{tabular}

Fuente: Souto, Benedito y Cervellera (2000).

15 En el año 1998 presentó su Trabajo de Investigación dentro del programa de doctorado: BENEDITO SIFRE, $\mathrm{M}^{\mathrm{a}}$ Teresa. Investigación didáctica de la geografía de la población, Trabajo inédito, que después sintetizó en un artículo conjunto: Souto, Benedito y Cervellera, (2000)

16 Utilizamos como referencia la editorial y el año de edición del manual escolar. 
Pese al diferente número de páginas de los manuales de EGB y de BUP, la síntesis informativa es la misma. Es decir, el texto de autor reproduce los mismos conceptos en un caso y otro, mientras que la explicación y las relaciones causa>consecuencia aumentan en el caso de BUP, como podemos ver en estos dos ejemplos (cuadro 4) que proceden de los libros de la editorial Santillana y Vicens Vives ${ }^{17}$ :

Cuadro 4: Análisis de texto de autor en manuales escolares

\begin{tabular}{|c|c|}
\hline Conceptos/consecuencias en EGB & Conceptos/consecuencias en BUP \\
\hline $\begin{array}{l}\text { Migración, emigrar, inmigrantes } \\
\text { Países o regiones que suministran emigrantes } \\
\text { Países que acogen inmigrantes }\end{array}$ & $\begin{array}{l}\text { Migraciones, emigración, inmigración, país } \\
\text { emisor, país receptor }\end{array}$ \\
\hline $\begin{array}{l}\text { Envejecimiento o rejuvenecimiento según el tipo } \\
\text { de movimiento (emigrar/inmigrar) }\end{array}$ & $\begin{array}{l}\text { Mayor número de consecuencias: demográficas, } \\
\text { sociales y económicas }\end{array}$ \\
\hline $\begin{array}{l}\text { Tasa bruta de mortalidad, tasa de mortalidad por } \\
\text { edades, tasa de mortalidad infantil, mortalidad y } \\
\text { desarrollo }\end{array}$ & $\begin{array}{l}\text { Tasa de mortalidad, tasa de mortalidad infantil, } \\
\text { factores en distintos países con grado de desarrollo } \\
\text { diferente }\end{array}$ \\
\hline
\end{tabular}

Fuente: Elaboración propia

En consecuencia podemos observar que los contenidos del texto de autor se refieren a listados conceptuales que explican algunas situaciones geodemográficas estereotipadas y que responden a modelos académicos. Por eso la dificultad que tienen los alumnos para su aprendizaje, pues no están relacionados los términos con problemas que ellos y ellas puedan percibir en su cotidianidad.

Para completar esta visión de la geografía de la población hemos recurrir al apartado de actividades y documentos, pues complementaban la visión "objetivista" del texto de autor. Un ejemplo significativo es el modelo del proyecto Didespai ${ }^{18}$, donde el problema básico que articula todo el contenido es el crecimiento de la población. Si bien se hace referencia a los condicionantes sociales, políticos y económicos, realmente los elementos que analizan son los que se corresponden con la dinámica demográfica estadística (crecimiento natural y saldo migratorio). Existe toda una serie de datos numéricos, gráficos y figuras para ilustrar cómo varía la población en un lugar determinado. Igual sucede con el proyecto de innovación realizado en el Instituto de Ciencias de la Educación del País Vasco ${ }^{19}$, que consiste en un proyecto de trabajos prácticos, en el cual no hay enlace teórico entre las diversas actividades.

17 Hemos seleccionado estas dos editoriales porque eran, en su momento, las más importantes por el número de ventas de material escolar.

18 DIDESPAI, Grupo. Geografía humana. Proyecto de renovación de la Didáctica de Geografía, Barcelona: Vicens-Vives, 1986

19 AUTORES VARIOS. Programación didáctica de la asignatura de $2^{\circ}$ de BUP: Geografía humana y económica, Bilbao: Universidad del País Vasco, 1984 


\section{UN ESTUDIO DE CASO: EL TRABAJO Y LA POBLACIÓN ACTIVA.}

El concepto de población activa, que forma parte de la enseñanza de la Geografía, define, en un país, las personas que se han integrado en el mercado de trabajo, es decir, que trabajan o que buscan trabajo. Esta conceptualización no sólo es escueta y poco concordante con la realidad, sino además, discriminadora. No incluye a todas aquellas personas que, no teniendo un empleo asalariado ni buscándolo, son clasificadas como "población no activa", dando un sentido peyorativo a su inactividad que, como decíamos, no responde a la realidad: hablamos del trabajo no remunerado, tanto de amas de casa, como de estudiantes y voluntarios, que contribuyen socialmente al crecimiento y mantenimiento de la población en tanto que inversión en aquellos que trabajan. Otorgar al trabajo únicamente un valor mercantil, descartando a todos aquellos que hacen de su trabajo una labor social y una inversión en el futuro de otros, constituye un sesgo pernicioso que no responde a la realidad social vivida por los alumnos.

La impugnación del concepto de población activa

Esta preocupación ha sido la que ha guiado una investigación académica que hemos realizado sobre el currículo real, ese que surge de la interacción que se produce en las aulas escolares entre el aprendizaje del alumnado, la enseñanza del profesorado y la codificación de los hechos sociales en los manuales escolares20. El estudio de casos, realizado en 2013 con casi 150 alumnos de $2^{\circ}$ y $3^{\circ}$ de ESO21 pertenecientes a centros de la provincia de Valencia (España), ha demostrado que este concepto puede y debe ser impugnado en la enseñanza de la Geografía escolar a través de la intervención del profesorado, la innovación educativa y la vivencia cotidiana de los alumnos.

El concepto de población activa en los manuales

La consideración del concepto de población activa la encontramos también en muchos manuales escolares (libros de texto) que emplean los alumnos españoles en el aula. Haremos una selección de cinco ejemplares 22 para hacer constar cómo es tratado el concepto de población activa en cada uno de ellos. En primer lugar haremos una relación de los libros de texto consultados, tal como se puede comprobar en el cuadro siguiente (ver cuadro 5), donde tenemos una síntesis de la muestra obtenida.

20 Nos referimos al Trabajo Fin de Máster de Investigación en didácticas específicas de Ana Maria RIBES CRESPO: La impugnación del concepto de población activa en la enseñanza de la Geografía, presentado en septiembre de 2013 en la Facultat de Magisteri de la Universitat de València con la máxima calificación.

21 La totalidad de los alumnos que participaron en el estudio de caso fue la siguiente: 54 alumnos en Tavernes Blanques, 36 alumnos en Picanya, 42 alumnos en Algemesí, 15 en Tavernes de la Valldigna.

22 Tres editoriales (Vicens, Anaya y Santillana) representan prácticamente el 50\% del total de manuales escolares del área y curso escolar que analizamos en el total de España. Las otras dos editoriales se han utilizado para contrastar y ver la homogeneidad de los planteamientos educativos respecto al análisis del mundo del trabajo 
Cuadro 5. Definición de población activa en manuales escolares

\begin{tabular}{|c|c|c|}
\hline Curso escolar & Manual escolar & Definición de población activa \\
\hline $\begin{array}{l}3^{\circ} \text { de ESO (14/15 años de } \\
\text { edad) }\end{array}$ & BURGOS ALONSO,1994 & $\begin{array}{l}\text { "Población activa: es la suma de la } \\
\text { población ocupada y la que busca } \\
\text { empleo". (pág. 226). }\end{array}$ \\
\hline $3^{\circ}$ de ESO & ARANDA BERNAL, 1995 & $\begin{array}{l}\text { "Un elemento que caracteriza a la } \\
\text { estructura de población es el que se da } \\
\text { conforme a su base socioeconómica, es } \\
\text { decir, el que divide la población en activa, } \\
\text { integrada por la que realiza una actividad } \\
\text { remunerada, e inactiva, la que consume } \\
\text { sin producir, aunque este término } \\
\text { actualmente es ambiguo y se encuentra en } \\
\text { revisión" (pág. 52). }\end{array}$ \\
\hline $3^{\circ}$ de ESO & ALBET MAS, 2011 & $\begin{array}{l}\text { "La población activa la integran todas las } \\
\text { personas ocupadas que se dedican a la } \\
\text { producción de bienes y de servicios u las } \\
\text { personas que están en el paro o que buscan } \\
\text { trabajo por primera vez" (pág. 32). }\end{array}$ \\
\hline $3^{\circ}$ de $\mathrm{ESO}$ & BURGOS ALONSO, 2012 & $\begin{array}{l}\text { "La actividad de la población permite } \\
\text { distinguir entre población activa, } \\
\text { que desempeña o busca un trabajo } \\
\text { remunerado, e incluye a la población } \\
\text { ocupada, o con trabajo, y a los parados y } \\
\text { personas que buscan su primer empleo; } \\
\text { y población inactiva, que no realiza una } \\
\text { actividad profesional remunerada, como } \\
\text { los estudiantes, los pensionistas y las } \\
\text { personas dedicadas a las tareas del hogar". } \\
\text { (pág. 240). }\end{array}$ \\
\hline $\begin{array}{l}2^{\circ} \text { de ESO (13/14 años de } \\
\text { edad) }\end{array}$ & $\begin{array}{l}\text { BRANDI FERNÁNDEZ, } \\
2012\end{array}$ & $\begin{array}{l}\text { "Población activa: personas de } 16 \text { años } \\
\text { o más que trabajan o están dispuestas } \\
\text { a hacerlo. Por lo tanto, es la suma de la } \\
\text { población ocupada y en paro" (pág. 311). } \\
\text { "Población activa femenina. Son las } \\
\text { mujeres que trabajan o están en edad y } \\
\text { disposición de trabajar. Se expresa en } \\
\text { porcentaje de la población femeninas } \\
\text { mayor de } 15 \text { años".(Documento 9, página } \\
\text { 264). }\end{array}$ \\
\hline
\end{tabular}

Fuente: Elaboración propia sobre manuales escolares 
Por lo que podemos ver a través de estos cinco manuales consultados, el concepto general de población activa excluye sistemáticamente a todas las personas que no pueden ser incluidas en esta estricta definición, lo que supone la discriminación u olvido de aquellos sectores que, realizando una actividad que equivale a un trabajo, un servicio o una producción, por el hecho de no ser remunerada y/o de no estar buscando una actividad remunerada, no pueden ser considerados población activa. Son tanto las amas de casa, como los estudiantes, jubilados y voluntarios, pero en esta investigación nos centraremos fundamentalmente en los dos primeros grupos y un poco más superficialmente en los segundos. Es una definición que supone un resumen conceptual y factual de las publicaciones académicas y estadísticas oficiales, sin cuestionarse si dichos hechos y conceptos aparecen en la vida cotidiana del alumnado.

Como se puede apreciar en la definición de población activa en estos cinco manuales, existe una tradición de considerar una división muy estricta entre trabajo y no trabajo, no considerando las situaciones de empleo a tiempo parcial, que actualmente es frecuente. Esto tiene un claro reflejo en la praxis docente, transmitiéndose a los alumnos esta concepción parcial. La investigación o estudio de caso que vamos a presentar pretende ser una alternativa a esta realidad.

La definición de la población activa en los alumnos

El estudio de casos realizado con alumnos de $2^{\circ}$ y $3^{\circ}$ de ESO de centros escolares en Picanya, Tavernes Blanques, Tavernes de la Valldigna y Algemesí, todos ellos ubicados en la provincia de Valencia (España), tenía como finalidad comprobar los conocimientos y opiniones de los alumnos al respecto y, por supuesto, impugnar la enseñanza actual de este concepto.

Dada la extensión de la investigación didáctica en sí, hemos seleccionado únicamente tres apartados que consideramos básicos para el propósito de este artículo: uno es una selección de una pregunta perteneciente a un cuestionario pasado a los alumnos de todos los centros con la intención de comprobar sus conocimientos y opiniones. La pregunta seleccionada es la tercera del primer apartado del cuestionario (P3)23, especificando las opciones disponibles, a escoger cuantas fueran verdaderas por el alumno: a) Trabajadores asalariados o en paro. b) Amas de casa. c) Jubilados. d) Estudiantes.

23 Asi mismo, las siglas visibles en el cuadro indican los códigos de los grupos que participaron en el estudio: Grupo Experimental Picanya 1 sería GEP1, Grupo Experimental Tavernes de la Valldigna sería GETV, Grupo Control Tavernes Blanques sería GCTB, Grupo Control Algemesí sería GCA, y así sucesivamente. 
Cuadro 6. Resultados del cuestionario realizado con el alumnado de los grupos de control y experimentales

$\begin{array}{lcccc}\text { P3: ¿Cuáles de estos grupos sociales son considerados población active } \\ & \text { A } & \text { B } & \text { C } & \text { D } \\ \text { GEP1 } & 12 & 3 & 0 & 3 \\ \text { GEP2 } & 14 & 0 & 0 & 4 \\ \text { GETV } & 10 & 2 & 2 & 7 \\ \text { GETB } & 14 & 2 & 0 & 3 \\ \text { GCTB } & 15 & 4 & 0 & 1 \\ \text { GCA } & 12 & 3 & 1 & 5\end{array}$

Fuente: Elaboración propia

Una vez que se había debatido en clase qué es trabajo, se pretendía con esta pregunta comprobar los recuerdos y percepciones de los alumnos acerca de qué es población activa, una pregunta decisiva para el objeto de la investigación. La inmensa mayoría de los alumnos encuestados, independientemente de si pertenecían a un grupo de control o experimental, han escogido la opción A (trabajadores asalariados o en paro) como respuesta, es decir, incluyendo indudablemente a este grupo de personas dentro de la población activa.

Esto nos hace pensar que los alumnos han recurrido a sus recuerdos y saberes procedentes de la percepción genérica y más habitual acerca de quiénes se incluyen en el grupo de la población activa. Por lo tanto, era lógico que la inmensa mayoría de los encuestados marcaran esta opción. Ello no nos permite conocer qué saben y no saben acerca del mundo del trabajo, sino que nos permite deducir que su conocimiento acerca de la población activa procede del conocimiento vulgar que se reproduce en el escolar. Por eso hemos comprobado que no hay diferencia entre los grupos experimentales y los de control, pues ambos reproducen una opinión superficial y simple de la realidad compleja del mundo laboral; tan sólo se refieren a la definición que agrupa a las personas por su condición en el mercado laboral. Es más, la totalidad de los alumnos encuestados (15) que ha marcado la opción A pertenecen, curiosamente, a un grupo control, el de Tavernes Blanques (GCTB), por lo que parece ser que en este caso los alumnos han apelado a su conocimiento de base y a lo aprendido en clase, independientemente de si han recibido innovación didáctica o no. Estas conclusiones nos hacen pensar que el conocimiento que se adquiere habitualmente en el sistema escolar no cuestiona los hechos sociales que se transmiten vulgarmente.

Las otras opciones marcadas lo son en cifras tan bajas que no permiten extraer una conclusión relevante. Por lo tanto, en este caso no se manifiesta diferencia alguna entre grupos experimentales o de control: el saber general se reafirma, el concepto de población activa engloba únicamente a los asalariados o en paro; los estudiantes, jubilados y amas de casa están excluidos. Ellos son conscientes de ello, lo saben, lo intuyen y así lo manifiestan. Hemos comprobado que en algunos casos la población en paro no figuraba dentro del concepto de población activa, como así sucede en una definición académica, pues se impone la condición de mercantilización de la vida laboral. En todo caso hemos comprobado que el alumnado es capaz de recordar la definición canónica de población activa, pero no matizar esta conceptualización. 
El estudio cualitativo del mundo laboral

Por eso fue necesario acudir a otra metodología de trabajo en el estudio, como fue la grabación en vídeo de debates en torno a estos conceptos en la sesión de aula. En este punto, hemos seleccionado dos breves extractos de los debates realizados en clase -grabados en vídeo- que nos permiten hacer notar cómo, tanto en el caso de los grupos experimentales como de control, los alumnos tendían a identificar a los parados como población no activa. Ello nos alertó de la dificultad de conceptualizar la población activa y los agentes laborales; es decir, aparentemente saben definir qué significa población activa, pero cuando deben comentar aspectos de su vida cotidiana no son capaces aplicar dichos conceptos a su realidad inmediata.

IES “La Valldigna” de Tavernes de la Valldigna. Quinta sesión: (14/03/2013) 2 ESO

Profesor: Ahí falta algo per subratllar, perquè si no dóna lloc a confusió. Anem a vore. ¿La població activa és només els que tenen treball?

Alumna1: No.

Profesor: Qui falta?

Alumna1: Els estudiants. (El profesor niega con la cabeza). Les ames de casa. (Vuelve a negar). Ja ho diré.

Profesor: Mireu el mapa conceptual que vaig fer jo en la pissarra.

Alumno1: Els jubilats!

Profesor: (Negando otra vez con la cabeza) La població activa són els que estan treballant i qui més?

Alumna1: Els aturats i desempleats.

Profesor: Molt bé. Anem a vore. Recordem que població activa no són només els que treballen, sinó que són també els que estan buscant treball, per primera vegada, o perquè els han tirat del treball, perquè estan aturats i aturades. Població activa inclou als dos. (...) Estan actius treballant o estan actius buscant.

IES "La Valldigna” de Tavernes de la Valldigna. Sexta sesión: (21/03/2013) 2 ESO

Profesor: Mireu la frase que està subratllada. (...) Eixa és important. (...) A vore, té un error. (...) A vore qui el pilla. (...) Diu: "Desde el Gobierno afirman que las cifras del paro se han contenido - s'han aguantat, no han crescut- desde principios de año (...) debido al aumento de la población activa”. (...) Hi ha un error. Què puja? Què li passa a la població activa? (...) Ha augmentat, no? La població activa ha augmentat. (...) I com que ha augmentat, l'atur, ha disminuit, no? Què composa la població activa? Quins dos grups? 
Alumno1: Els que estan treballant i els que no.

Profesor: Ocupats, sí o no? I els aturats, no? Els dos són població activa, no? Aleshores, si la població activa ha augmentat...

Alumna1: Pues hi ha més ocupats i més desocupats.

Profesor: En principi hi han més ocupats i més aturats. Aleshores... jo podria dir eixa frase? Que "l'atur ha disminuit perquè ha augmentat la població activa"? És correcta?

Alumnos: No.

Alumna1: Si la població puja, puja tot.

Profesor: Aleshores, què estan confonent ahí? Estan confonent dos conceptes. Per a ells, la població activa, amb qui la confonen?

Alumna1: Amb els ocupats.

Profesor: Amb els ocupats. Ells fan, el que es escriu la notícia, identifica "activa" amb "ocupats". Això es fals. Els actius no són només els ocupats, són també els aturats.

Alumno1: Ah, ja ho entenc.

Professor: Està clar o no? El o la qui ha escrit això, ha clavat la pota.

En general es necesaria la intervención del profesor para guiar a los alumnos a la hora de definir qué es población activa. Para ellos es un concepto extraño y necesitan ser introducidos en el mismo, primero, definiéndolos según el saber epistemológico oficial de la Geografía, después, motivando en ellos la aportación de sus apreciaciones personales. En general, una vez se les ha guiado adecuadamente para que clarifiquen los conceptos y valoren personalmente la pertinencia de los mismos, los alumnos comprenden fácilmente el significado "oficial" de lo que es población activa y trabajo, y pueden llegar a constatar personalmente las contradicciones y divergencias entre el saber académico y epistemológico y la realidad social cotidiana en la que se mueven cada día.

Como la extensión de los testimonios de este estudio de caso es enorme, hemos decidido seleccionar para este artículo, por último, algunas intervenciones destacadas de los alumnos que han quedado reflejadas en sus cuadernos a partir del trabajo del dossier BOSEVA (Proyecto de Educación Geográfica Iberoamericano) que fue el material de base de dicha investigación. Naturalmente y debido al interés que nos centra en este artículo, tan sólo hemos seleccionado las intervenciones más destacadas de la categoría correspondiente al concepto de población activa y trabajo, y las respuestas de los alumnos a cuestiones concretas del dossier trabajado en clase.

¿A quiénes discrimina el concepto de población activa? ( $2^{\mathrm{a}}$ sesión)

Esta pregunta pretendía hacer una recapitulación sobre lo trabajado en clase; en concreto, que 
los alumnos expresaran su opinión y reflexionaran sobre el alcance del concepto de población activa y especialmente, al dilucidar sobre quiénes están incluidos, razonar sobre a quiénes excluye. En última instancia, se proponía hacer notar a los alumnos quiénes son los excluidos de la coyuntura del mercado laboral (los parados, por ejemplo). Tanto en este caso como en los demás, hemos seleccionado únicamente las intervenciones más destacadas.

1FTB24: "Discrimina a las amas de casa, a los estudiantes, los niños y los jubilados, porque los muestran en el esquema como que no realizan ninguna función. Los discriminan a ellos porque dicen o los muestran como que no realizan ninguna función activa, para mí los discriminan por no tener trabajo".

2FTB: "Discrimina a amas de casa, jubilados, pensionistas, estudiantes, menores que esa es la población no activa y la discrimina porque piensan que no hacen lo mismo pero sin embargo hacen lo mismo pero sin cobrar."

3FTB: "A estudiantes, menores de 16, a jubilados, a pensionistas que no trabajan, a parados. Lo hacen porque piensan que no hacen ninguna actividad pero si que hacen actividades, se piensan que no hacen nada".

2FP: "A las amas de casa, porque trabajan, pero la gente no lo considera un trabajo".

¿Por qué se clasifica a las personas según su actividad y condición en el mercado laboral? ( $2^{\mathrm{a}}$ sesión)

Esta pregunta pretendía comprobar los conocimientos de los alumnos respecto a la clasificación del mercado laboral -sectores primario, secundario y terciario; activas y no activas, paradas, jubiladas, etc.- y recapitular sobre algunos debates y observaciones vertidas en las sesiones de aula. En particular, lo que reveló estas respuestas fue que los alumnos no tenían nada clara la razón de esta clasificación. Unos atribuyen esta clasificación a un mero control social y gubernamental, otros lo interpretan como una especie de estadística, de base de datos consultable; e incluso una jerarquía de mejores y peores trabajos en función de formación y sueldo. No hubo muchas opiniones unánimes y, en general, no comprendieron el porqué de esta clasificación.

2FTB: "Se clasifican para saber cuántas personas están activas y cuántas no lo están y si realizan alguna activad o no".

3FTB: "Porque así se tiene más organizado todo y se puede saber a que se dedica la gente y hacer diferentes porcentajes".

3MTB: "Porque es una buena manera de distinguir las distintas clases de situaciones sociales en este contexto".

24 Nuevamente, los códigos expresan el orden de intervención, sexo y pertenencia a un grupo concreto del alumno cuya anotación se ha destacado. Así, 1FTB es la primera alumna del grupo de Tavernes Blanques y 2FP, por ejemplo, sería la segunda alumna del grupo de Picanya. En este caso todos son pertenecientes a grupos Experimentales, pues sólo éstos trabajaron con el dossier BOSEVA. 
6FTB: "Para saber la cantidad de jente que hay desempleada y con empleo y saber en qué condición trabaja".

2MP: "Porque hay gente mas preparada para un oficio que otra, por lo tanto seria mas lógico darle a esos el trabajo."

2FP: "Porque cuando mas cualifido este una persona, mejor trabajo tiene".

3MP: "Para tener a cada persona controlada en un sector".

3FP: "Porque según esto, las personas cobran mas o menos y tienen trabajo, mejores o peores y asi se sabe cuales son mejores y mas importantes".

¿El trabajo es un valor humano o un simple precio mercantil? ( $2^{\mathrm{a}}$ sesión)

Esta pregunta entrañaba cierta complejidad, en tanto en cuanto no se había trabajado directamente en el aula la resolución de una cuestión como ésta. Pretendía que los alumnos, al cabo de todo lo reflexionado, estudiado y trabajado en clase, llegaran a una valoración personal de cómo está valorado el trabajo en la sociedad: cuál es su valor real y cuál debería ser. Aunque las respuestas fueron muy ricas y se pudieron apreciar razonamientos muy interesantes, por lo tanto, demostrando que los alumnos son conscientes de que el trabajo es algo más que un precio mercantil, sin embargo no saben qué valor humano es el que tiene.

1FTB: "En mi opinión el trabajo es un valor humano, ya que un estudiante que estudia, ese estudio que hace es un trabajo, o cuando tú vas a cuidar de tus primos o de tu hermano. También se puede decir que es un valor humano ya que tú estás realizando una función".

2FTB: "Pienso que es un simple precio mercantil, ya que si fuéramos ricos no necesitaríamos trabajar para ganarnos la vida".

3FTB: "También es un valor humano porque con un trabajo te sientes más seguro y adaptado a la sociedad".

9FTB: "Según el valor humano es que trabajas por amor y el precio mercantil que trabajas por dinero".

2MTB: "Un precio mercantil, porque yo creo que si no pagaran por trabajar, la mayoría no trabajaría".

11FTB: "Las dos cosas, para ganar dinero para vivir y para sentirse útil en la vida".

1FTV: "És un valor humano porque la persona quiere trabajar y ser algo, y también es un precio mercantil, porque ganan dinero y les hace falta para poder vivir".

1MTV: "Valor humano porque cada persona cumple un papel desde el estudiante a la ama de casa". 
1FP: "Es un valor humano peo a la vez tanbién hay veces que es un precio mercantil, como ocurre en algunos trabajos donde no se necesita ninguna qualificación".

4MP: "Las 2 cosas por una parte te llena de valor humano porque te sientes util i por otra parte mercantil por su parte de ventas".

Para cerrar este apartado, podemos concluir que el concepto de población activa, tal y como ha quedado reflejado en la dinámica de clase y en los materiales generados por los alumnos durante esta investigación, no aclara, sino dificulta, el estudio de los problemas humanos. Se trata de un concepto básico de geografía que no ayuda a entender los problemas en relación al mercado laboral ni las condiciones del trabajo, puesto que genera confusión entre los alumnos, al no responder a la realidad cotidiana que ellos viven. Se genera, por tanto, una distorsión entre realidad social y saber conceptual, que es necesario paliar si queremos que los alumnos adquieran una capacidad crítica para la realidad que les rodea. Distorsión que viene asociada a una vertiente técnica de la enseñanza de la geografía que prevalece sobre la metodológica: se aportan muchos datos numéricos en los materiales didácticos habituales -libros de texto- pero no se plantea una enseñanza-aprendizaje por problemas. Esto lleva a que, realmente, se planteen y reflexionen pocos problemas geográficos relevantes en el marco del aula, siendo la única excepción el fenómeno del exceso demográfico.

Se hace necesario, pues, trabajar por problemas en el aula, porque esta carencia de resolución de problemas en el marco de la enseñanza de la geografía es un problema que se arrastra desde hace mucho tiempo, y por más que se hacen observaciones y estudios sobre esta realidad, no hay progresos reales en la praxis docente ni en el currículum presente. Esto se debe, fundamentalmente, al anclaje de los profesores a los libros de texto, libros de texto que son fruto de editoriales que no tienen ningún tipo de compromiso con cuestiones de innovación didáctica.

\section{CONCLUSIONES}

Los estudios de geografía de la población han reflejado la tendencia de presentar la dinámica y estructura demográfica como un hecho natural, sin cuestionarse la problemática social que afecta a las personas; algunas muy cercanas a los sentimientos y emociones del propio alumnado.

El estudio del currículo oficial y de los manuales escolares en España (que se puede generalizar a otros países iberoamericanos) revela una concepción de la geografía muy cercana a algunas de las preocupaciones académicas 25 . Sin embargo, dicha transposición se hace de forma superficial y en dos elementos yuxtapuestos (conceptos y técnicas) que obedecen a una concepción educativa, sea ésta implícita o poco reflexiva. Es decir, se busca la legitimidad de la innovación en los hechos conceptuales y no tanto en la manera de cuestionarse la realidad.

Por una parte aparecen las definiciones conceptuales en los textos de autor, que se corresponde

25 Hemos consultado la información de la página web (http://www.agepoblacion.org/) del Grupo de Población de la Asociación de Geógrafos Españoles (AGE) en la cual hemos consultado el Boletín de Noticias y el informe del año 2013 donde se puede comprobar la variedad de proyectos de investigación en relación con problemas de envejecimiento demográfico, movilidad de la población, planificación urbana o interculturalidad. 
con un reflejo de la cultura distinguida, o sea, aquello que debe aprender el alumnado. Por otra aparecen las técnicas estadísticas y gráficas como una traducción simplista de la metodología activa, que se presenta como una manipulación de los documentos y datos, generalmente demográficos. Es decir, no se trata de instrumentos necesarios para resolver problemas sociales, sino de tareas instrumentales con una finalidad en sí mismas.

En el estudio de caso, de la geografía de la población activa y el análisis del ámbito geográfico del trabajo humano, hemos podido constatar que el alumnado a través de sucesivos debates y bajo la dirección pedagógica del profesorado, ha podido integrar sus preocupaciones diarias en una explicación conceptual de los problemas sociales. Y para ello ha sido de gran ayuda la elaboración de unos materiales específicos, con su metodología didáctica. Ha sido el caso del proyecto BOSEVA, que se ha experimentado en Bogotá (Colombia), La Serena (Chile) y Valencia (España). Con ello alumnos y docentes han impugnado un concepto ambiguo y poco preciso, como es el de la población activa, lo que ha permitido que afloren situaciones de la vida cotidiana en la cual existen marginaciones sociales, precarización laboral y discriminación de género. Y dicha propuesta nos ha permitido visualizar una alternativa en la programación de actividades para estudiar el mundo laboral (ver figuras 1 y 2), Como se puede comprobar a través de nuestra alternativa metodológica procuramos organizar los conceptos de una forma más dinámica, para de esta forma abordar los problemas del trabajo cotidiano.

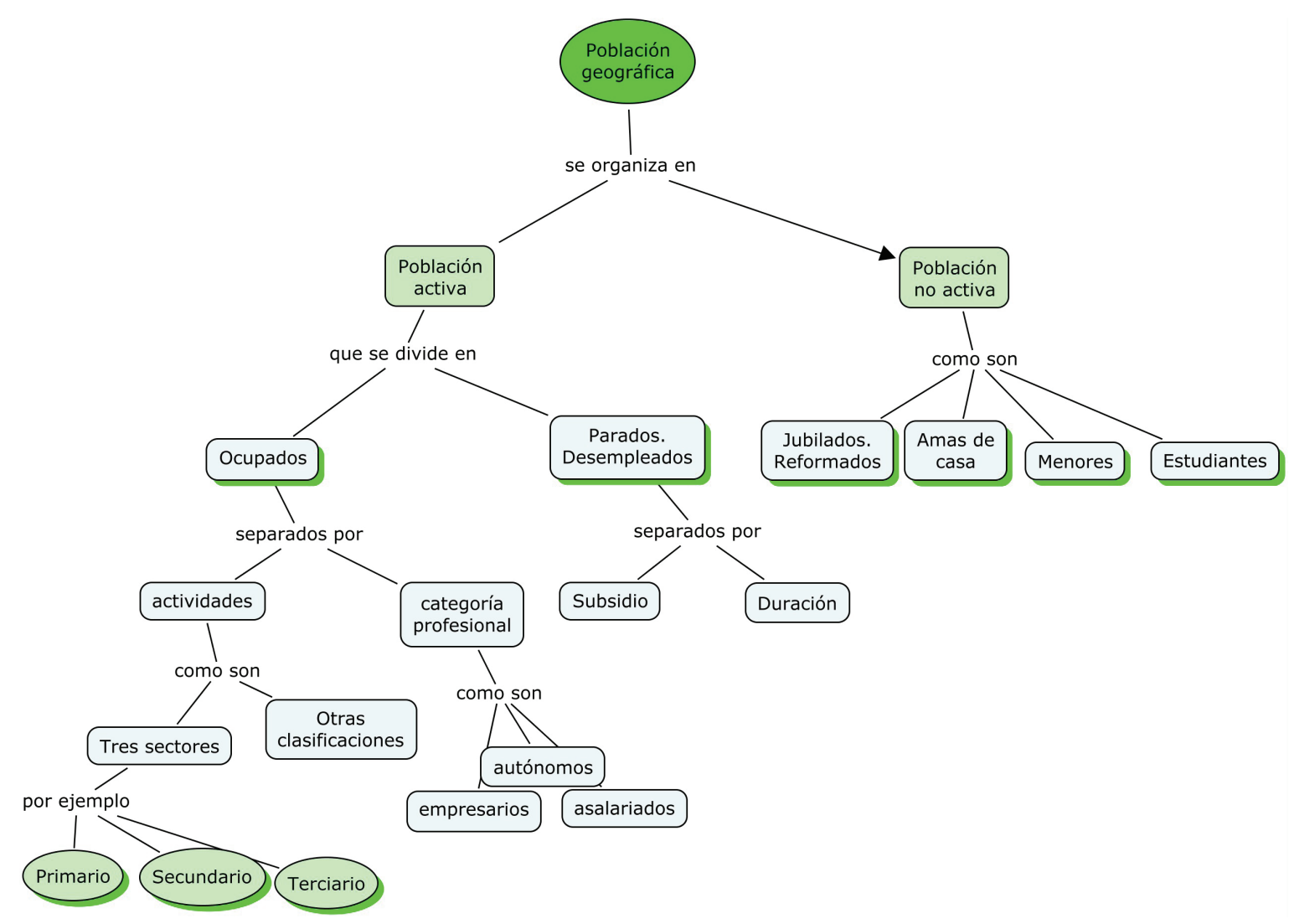

Figura 1.- Conceptos básicos de la geografía de la población activa 


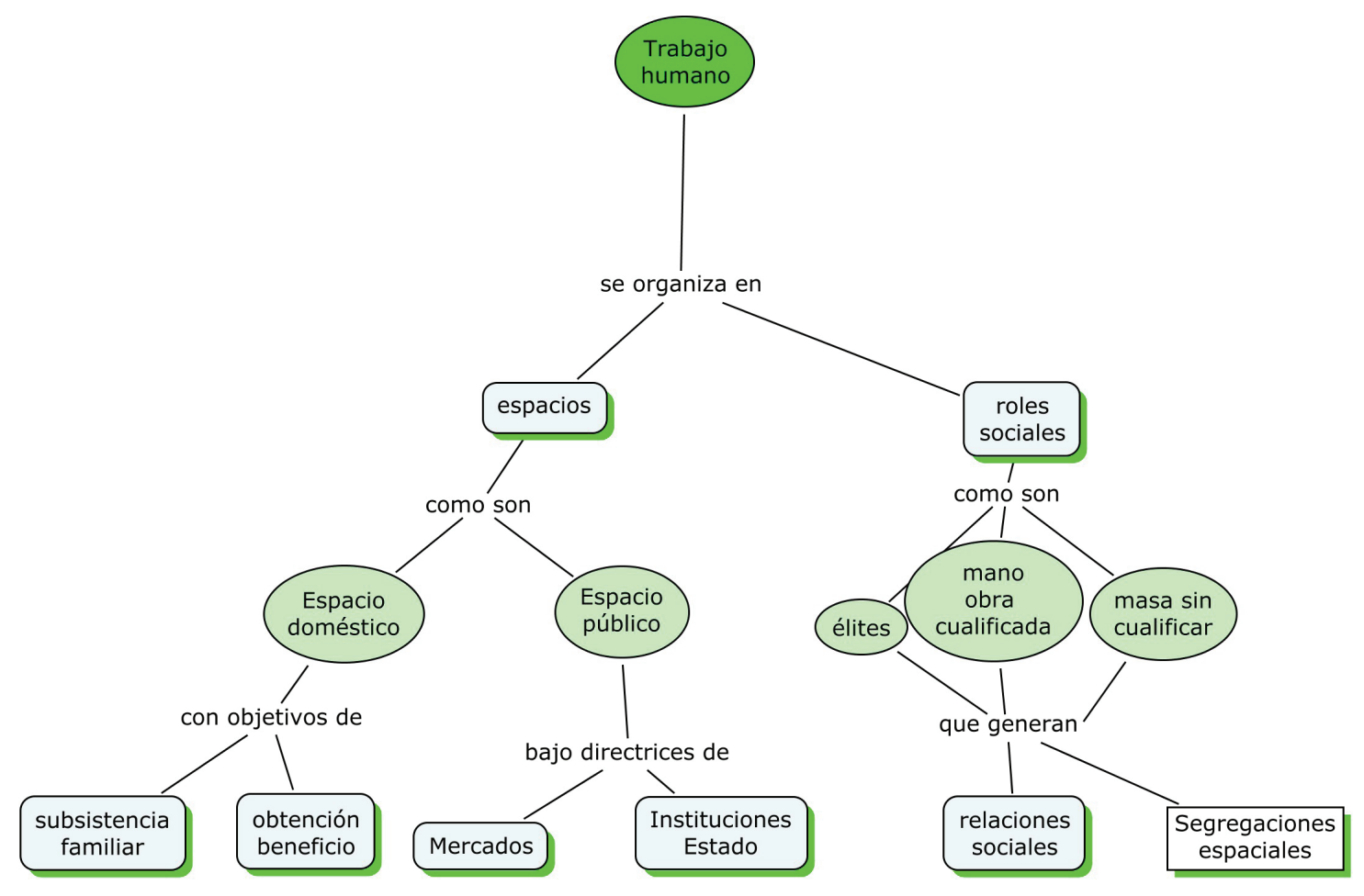

Figura 2. Nuevos conceptos que aparecen en el análisis del mundo laboral

Con esta aportación de análisis de caso hemos querido mostrar cómo desde una reflexión teórica sobre la evolución de la geografía de la población que se enseña en la educación básica es posible descubrir los obstáculos que hacen poco significativo el aprendizaje de los hechos geográficos en las aulas escolares. Una cuestión que afecta decisivamente a la representación social de la materia, como es el caso de la vulgarización del conocimiento de la geografía.

\section{REFERENCIAS}

\section{A. RELACIÓN DE MANUALES ESCOLARES CONSULTADOS}

Albet Mas, A; Benejam Arguimbau, P; Casa Vilalta, M; Comas Solé, P; Oller Freixa, M; Nou Àgora. Ciències Socials, Geografia. Comunitat Valenciana. Ed. Vicens Vives, Madrid 2011.

Alvares Oses, José A.; Sabán Gutiérrez, A.; Martín Redondo, G. Geografia humana y económica, Madrid: Santillana, 1987

Aranda Bernal, A. M; León Sánchez, I; Ruiz González, J, Ferrer Fortuny, R; Mateo Núñez, R. Geografía. Ciencias Sociales Segundo Ciclo. Educación Secundaria Obligatoria. Comunidad Valenciana. Ed. McGraw Hill, Madrid 1995.

Asián Peña, José Luis E Ibáñez Daltabuit, Isabel. Geografia General, Barcelona: Bosch, 1968. 
Autores Varios. Programación didáctica de la asignatura de $2^{\circ}$ de BUP: Geografia humana y económica, Bilbao: Universidad del País Vasco, 1984

Benejam, Pilar et al. Intercanvi. Geografia humana i econòmica del món actual, Barcelona: Vicens-Vives, 1983

Borrás, M. Etnos. Ciencias sociales, $6^{\circ} E G B$, Barcelona: Barcanova, 1983.

Burgos Alonso, M. (Coord.); Armesto Sánchez, J; Cosao Moyano, J; Saramillo Cervilla, M; Martín Guerrero, S; Cruz Villalón, J; Geografia General Proyecto 2000. Ciencias Sociales, Geografía e Historia. Educación Secundaria Obligatoria, 2 Ciclo. Ed. Algaida, Sevilla 1994.

Burgos Alonso, M; Muñoz-Delgado, M.c; Ciencias Sociales, Geografia e Historia. Comunidad Valenciana. Educación Secundaria 2. Unidades 10 a 12. Ed. Grupo Anaya, Madrid 2012.

Brandi Fernández, A; Gregori Soldevila, I; Geografia e Historia $2^{\circ}$ de ESO. Comunidad Valenciana. Proyecto Los Caminos del Saber. Ed. Voramar Santillana, Valencia 2012.

Comas De Montáñez, María. Geografia de España, Barcelona: Ediciones Sócrates, 1968.

Didespai, Grupo. Geografía humana. Proyecto de renovación de la Didáctica de Geografía, Barcelona: Vicens-Vives, 1986

Edetania, Grupo. Geografia Humana y Económica, Valencia. Editorial ECIR, 1986.

Edetania, Grupo. Cuaderno de actividades, Valencia: ECIR, 2005

Fenosa Tatay, X et al. Llibreta de Competències bàsiques, Segon curs. Barcelona: Vicens Vives, 2010.

Fernández, A. et. Ciencias sociales, $6^{\circ} E G B$, Barcelona: Vicens-Vives, 1988.

Garbí, Grup. Geografía Humana y Económica, Valencia: Consorci d'editors valencians, S.A., 1984.

Grima, Juan M. Geografía de España, Valencia: ECIR, López Mezquida Editor, 1960, 2ª edición 1963

Molinero, F; Méndez, R.; Alario, M. Secundaria. Geografia, Eso 2º ciclo, Madrid: Santillana, 1998

Perales, Josué Y Pelegero, Vicent. Ciencias sociales, $6^{\circ}$ EGB, Madrid: Anaya 1990

Rastrilla Pérez, Juan; Zubia, Antonio Y García Ruiz, Enrique. Geografia de España. Primer curso de Bachillerato, Madrid S.M. 1968 (en coedición con Bibliográfica Española, Bruño, L. Vives)

Vergés, Oriol. Sociedad. Fichas de trabajo, $8^{\circ}$ de Educación General Básica, Barcelona: Teide, 1974. 


\section{B. REFERENCIAS BIBLIOGRÁFICAS CITADAS EN EL TEXTO}

Giménez Hurtado, Vicenta P.; Souto González, Xosé M. (1993). La Geografia y los estudios de la población. Buenas intenciones y muchos números, Aula de Innovación Educativa, núm. 19; pp. 22-29

Izquierdo, A. (director) (2003). Inmigración: mercado de trabajo y protección social en España, Madrid: Consejo Económico y Social.

Puyol, R (1996). La población española, Madrid: Síntesis.

Puyol, R. (ed.) (1997). Dinámica de la población en España. Cambios demográficos en el último cuarto del siglo XX. Madrid, Síntesis.

Souto González, X.m., Benedito Sifre, M ${ }^{\mathrm{a}} \mathrm{T}$, Cervellera Martínez, A. (2000): Los manuales escolares y la didáctica de la geografia entre 1950 y 1990, Tiana Ferrer, A. (comp.) El libro escolar, reflejo de intenciones políticas e influencias pedagógicas, Madrid, UNED, pp. 63-85.

Articulo recibido en mayo 30 de 2013 - Aprobado en junio 30 de 2013 